\title{
Les théories des migrations. Synthèse de la prise de décision individuelle
}

Theories of Migration. Synthesis of Individual Decision-making

Las teorías de las migraciones. Síntesis de la toma de decisiones individual

\section{Étienne Piguet}

\section{(2) OpenEdition}

Journals

\section{Édition électronique}

URL : https://journals.openedition.org/remi/6571

DOI : 10.4000/remi.6571

ISSN : $1777-5418$

Éditeur

Université de Poitiers

Édition imprimée

Date de publication : 1 septembre 2013

Pagination : 141-161

ISBN : 979-10-90426-09-2

ISSN : 0765-0752

\section{Référence électronique}

Étienne Piguet, «Les théories des migrations. Synthèse de la prise de décision individuelle », Revue européenne des migrations internationales [En ligne], vol. 29 - n³ | 2013, mis en ligne le 01 septembre 2016, consulté le 14 avril 2022. URL : http://journals.openedition.org/remi/6571 ; DOI : https://doi.org/ 10.4000/remi.6571 


\section{Les théories des migrations. Synthèse de la prise de décision individuelle}

\section{Étienne Piguet ${ }^{1}$}

\section{Introduction}

Pourquoi migre-t-on ? Les chercheurs en sciences sociales tentent depuis plus d'un siècle de clarifier cette question qui concerne aussi bien la géographie que la psychologie, l'économie, la sociologie, l'anthropologie ou encore la démographie. Nous procédons dans les lignes qui suivent à une synthèse de ces réflexions théoriques. Nous brossons un tableau d'ensemble, sans entrer dans le détail des différentes approches, mais en mentionnant les principaux travaux de référence. Seule la théorisation de la migration en tant que déplacement dans l'espace est abordée ici, les théories de l'intégration et plus largement des conséquences de la migration ne sont pas abordées. Nous nous concentrons en outre sur l'échelle individuelle en mettant l'accent sur la prise de décision migratoire et ses facteurs explicatifs. Les théories macro orientées vers les flux agrégés (World system theory, théories de la globalisation, etc.) pourraient faire l'objet d'un autre article.

Cette synthèse ${ }^{2}$ est destinée principalement aux étudiants et aux chercheurs qui abordent la question des flux migratoires. Elle trouve sa raison d'être dans le fait que les synthèses existantes, tant en français (Tapinos, 1974 ; Courgeau et Lelièvre, 2003 ; Zlotnik, 2003 ; Noin, 2005) qu'en anglais (Zolberg, 1989 ; Cohen, 1996 ; Ghatak et al., 1996 ; Portes et Böröcz, 1996 ; Boyle et al., 1998 ; Arango, 2000 ; Geyer, 2002 ; Brettell et Hollifield, 2007) - et tout particulièrement la synthèse de référence de Massey et al. $(1993 ; 1994)$ - datent de plusieurs années et se concentrent généralement sur certaines disciplines seulement.

\footnotetext{
1 Professeur de géographie des mobilités, Université de Neuchâtel, Institut de géographie, Espace Louis-Agassiz 1, 2000 Neuchâtel, Suisse ; Etienne.piguet@unine.ch

2 Cette synthèse a été financée par le Swiss network for international studies (http:// www.snis.ch/) et I'Université de Neuchâtel dans le cadre du projet " Staying or Leaving? Migration in the Life Plans of Western African Students ". Une partie de l'article a été rédigée en collaboration avec G. De Coulon. Une version préliminaire a été publiée en tant que "working paper " (http://www2.unine.ch/maps/page28639.htmlhttp://doc.rero. ch/Im.php?url=1000,44,4,20101213163357-TM/Piguet_Etienne_-_La_volont_de_migrer_tat_ des_lieux_th_orique_20101213.pdf).
} 


\section{Les approches « classiques " de l'étude des migrations}

Ce premier courant est le plus ancien, il se fonde sur l'hypothèse centrale de rationalité et $\mathrm{d}^{\prime}$ homoeconomicus à laquelle sont apportées progressivement des complexifications. L'unité de référence reste l'individu, éventuellement la famille, sans que le contexte social soit très présent dans l'analyse.

\section{Le courant économique néoclassique}

Les prémisses d'un modèle théorique de décision migratoire peuvent déjà être identifiées chez Adam Smith (1776) et chez Friederich Ratzel (1882) mais on s'accorde souvent à considérer les " lois " du géographe Ernst Georg Ravenstein comme la première tentative explicite de théoriser les causes des migrations sur la base de l'observation des migrations internes au Royaume-Uni et des migrations internationales entre dix-neuf pays du monde. Dans ce contexte, Ravenstein mentionne de nombreux facteurs comme " produisant ou ayant produit des migrations ": de " mauvaises "lois ou des lois oppressives, des impôts élevés, un environnement social défavorable (uncongenial social surroundings), des événements forçant à migrer (" compulsion "), un climat peu attractif, etc. Le facteur selon lui le plus important tient cependant aux motivations économiques des acteurs (Ravenstein, 1889 : 286). Selon I'approche néoclassique issue de ces prémisses, la migration est une action rationnelle qui amène à maximiser l' " utilité ". Les acteurs comparent la satisfaction qu'ils retirent de leur localisation actuelle avec celle qu'ils pourraient retirer d'un déplacement et une insatisfaction résidentielle engendre un mouvement (Rossi, 1955). Pour Gerald Leslie et Arthur Richardson, la migration apparaît ainsi comme une stratégie parmi d'autres (changement d'emploi, choix familiaux, etc.) permettant à l'individu de réagir à une insatisfaction (Leslie et Richardson, 1961). Les acteurs sont considérés comme accordant principalement une "utilité " aux biens et services qu'ils peuvent acquérir et les facteurs explicatifs d'une migration sont dès lors approximés par les différentiels de salaires. L'agrégation des décisions individuelles explique ensuite les mouvements migratoires. Une propriété centrale du modèle néoclassique est qu'il considère la migration comme un facteur d'équilibrage des différences géographiques puisque les migrants vont se diriger des zones à bas salaires vers les zones à hauts salaires et vont dès lors modifier l'offre et la demande de travail.

Les économistes John Harris et Michael Todaro (1970) ont formalisé et approfondi ces idées de choix rationnel dans l'étude de l'exode rural. Selon leur modèle, ce n'est pas uniquement la différence de salaire entre deux espaces qui amène les personnes à migrer, mais le salaire espéré par le migrant potentiel, compte tenu de son profil et des coûts liés au déplacement. Ils introduisent dès lors dans le modèle une dimension probabiliste (les chances de trouver un emploi dépendent, entre autres, du taux de chômage) et des caractéristiques individuelles (les acteurs se distinguent par leur propension à accepter un certain risque, par l'utilité et le coût qu'ils associent à la migration et par des niveaux d'information inégaux). II en découle l'importante notion de sélectivité de la migration : des conditions structurelles identiques peuvent avoir un impact différent selon les individus ou les groupes (Massey et al., 1993; Chiswick, 2007). Ainsi les célibataires migrent souvent plus que les personnes ayant charge de famille car ils apprécient différemment les risques de la migration, etc. 
L'idée de "préférence pour le présent " caractéristique des hypothèses de l'économie néo-classique est aussi introduite dans le modèle sous la forme d'un taux d'escompte appliqué aux revenus futurs de la migration. Les néoclassiques envisagent en effet la possibilité d'une diminution de salaire durant une première période d'adaptation, compensée par une hausse de salaire plus marquée par la suite. S'il envisage ce scénario, un individu va décider de migrer, à moins qu'il ne suive l'adage " un tiens vaut mieux que deux tu l'auras " en appliquant au salaire futur un taux d'escompte élevé.

\section{Du choix rationnel à la rationalité limitée}

La théorie du choix rationnel (TCR) a été développée dans le cadre de l'économie puis reprise par des sociologues, dont un pionnier fut George Homans (1961). En tant que théorie de l'action, cette conception sous-tend une large part des théories migratoires et en particulier la vision néo-classique : les individus sont vus comme des acteurs qui choisissent au mieux de leurs intérêts entre des alternatives, même si des contraintes et des structures restreignent les choix possibles (Haug, 2008). La recherche a néanmoins ultérieurement complexifié I'image d'une personne migrante rationnelle qui chercherait uniquement à maximiser son utilité. Julian Wolpert (1965) décrit ainsi un acteur qui chercherait à " satisfaire " un besoin et non pas à le " maximiser ". La recherche d'une destination migratoire prendrait ainsi fin une fois une destination acceptable trouvée, sans nécessairement que l'ensemble des destinations possibles soient examinées. Les fondements théoriques de cet élargissement conceptuel renvoient à la notion de " rationalité limitée " (bounded rationality) développée quelques années auparavant par Herbert Simon (1955; 1957). Ils ouvrent la voie à la prise en compte de nombreux facteurs non directement liés au salaire dans la théorie des migrations et à l'idée d'un processus de prise de décision qui s'étale sur une certaine durée et implique un certain nombre d'étapes.

\section{Le cycle de vie}

Bien qu'elle se base sur les mêmes postulats que l'approche néo-classique, la prise en compte des cycles de vie introduit une dimension supplémentaire dans l'analyse en postulant que, suivant l'étape de son existence dans laquelle l'acteur se trouve, il aura plus ou moins de propension à migrer (Rossi, 1955 ; Leslie et Richardson, 1961). Ainsi, des personnes en début de carrière professionnelle et sans charge familiale seront plus mobiles. De même, une famille aura une plus forte propension à migrer avant la scolarisation des enfants, etc. Cette idée - aussi évoquée par Lee dans son fameux modèle push-pull des migrations (Lee, 1966) - sera reprise dans de nombreuses études relevant de plusieurs disciplines (De Jong et Gardner, 1981 ; Harbinson, 1981 ; De Jong et al., 1985). Dès les années 1970, la notion étroite et individuelle de cycle de vie sera cependant fortement critiquée en raison de sa restriction aux familles nucléaires, aux mariages stables et donc aux formes de cohabitation les plus traditionnelles (Uhlenberg, 1973 ; Boyle et al., 1998). On lui préfère désormais la notion, plus souple, de parcours de vie (Rérat et al., 2008 : 2), mais l'idée générale demeure celle d'une importance des caractéristiques démographiques individuelles dans l'analyse de la mobilité. 


\section{Le capital humain}

On définit généralement le capital humain comme constitué des compétences, des expériences et des savoirs qui permettent à l'individu d'acquérir un certain revenu par son travail. Cette idée, appliquée aux migrations dès les années 1960 (Sjaastad, 1962), a deux implications centrales. D'une part, le niveau et les caractéristiques du capital humain ont une influence sur la propension à migrer (ainsi une personne hautement qualifiée peut souvent plus facilement tirer parti de la migration et certaines formations - en informatique par exemple - sont plus facilement transférables à l'étranger que d'autres - en droit national par exemple). D'autre part, la migration peut, en elle-même, constituer une stratégie d'accroissement du capital humain (ainsi un séjour à l'étranger peut permettre d'acquérir un diplôme ou une expérience valorisables lors du retour au pays). Le capital humain a, par ailleurs, une influence sur l'attitude face à la migration : Everett Lee remarque, déjà en 1966, que plus une personne est formée, plus elle a de propension à prendre des risques, et donc à migrer. On peut cependant relever que la valorisation du capital humain s'avère parfois difficile et que le migrant disposant d'un capital élevé peut être confronté à une dévaluation professionnelle (Odland, 1988).

La notion de capital humain renforce la nécessité, déjà corrélative de celle de cycle de vie, d'adopter une perspective longitudinale sur la migration : les coûts et bénéfices sont estimés par le migrant potentiel non pas en comparant la période qui précède et qui suit la migration mais en considérant plusieurs années, voire la vie entière (Da Vanzo, 1981). Selon Siegfried Berninghaus et Hans-Günther Seifert-Vogt : "The Human Capital approach proved to be superior to the wage differential hypothesis, dominating till the sixties, which stated that migration decisions mainly depend on actual real wage differences between the countries. Compared with this, in Sjaastad's Human Capital model, migration decisions depend on actual and on future real wage differences (and migration costs) as well » (Berninghaus et Seifert-Vogt, 1987 : 201-202). La différence entre cette approche et la simple application d'un taux d'escompte (cf. supra) réside dans le fait que le migrant est ici à même d'avoir une certaine influence sur son futur en mettant en adéquation capital humain et choix de mobilité.

\section{L'information incomplète}

Une faiblesse aujourd'hui reconnue des approches décrites jusqu'ici réside dans la non-prise en compte du haut degré d'incertitude auquel doit faire face le migrant (Allen et Eaton, 2005). II ignore en effet souvent la valeur et la transférabilité de ses compétences et plus généralement la qualité de vie qui l'attend ailleurs. Ces informations s'avèrent pourtant essentielles au mécanisme de prise de décision qu'implique le modèle néo-classique. Ce problème, déjà signalé dans la littérature économique des années 1960 (Sjaastad, 1962), a été particulièrement approfondi par les géographes comportementalistes (Wolpert, 1965). Ces derniers ont cherché à déterminer les causes spatiales de la " connaissance imparfaite de l'information ", au premier rang desquelles a été placé le "frein de la distance ". D'autres chercheurs relativisent cependant l'effet de frein de la distance dans un contexte contemporain de circulation de plus en plus massive de l'information (Allen et Eaton, 2005). L'histoire migratoire individuelle prend de l'importance dans ce contexte puisque le fait pour un migrant de choisir 
un endroit où il se serait déjà rendu l'aide à bénéficier d'une information de qualité. La migration prend ainsi un caractère auto-entretenu ou cumulatif. Des recherches récentes identifient d'ailleurs l'expérience migratoire comme une composante du capital humain au même titre que la formation professionnelle sous le label de compétences circulatoires, de " savoir circuler " (Tarrius, 1989 ; Ma Mung et al., 1998 ; Berthomière et Hily, 2006). II en découle que les personnes ayant déjà une expérience migratoire sont plus susceptibles de formuler l'intention de migrer à nouveau (Fischer et al., 1997). Inversement, l'immobilité s'expliquerait partiellement par le coût de l'information lorsque le migrant potentiel n'y a pas un accès aisé. Certains chercheurs s'écartent cependant de l'équation " moins/plus d'information = moins/plus de migration " et postulent des effets contre intuitifs. Loin de freiner la migration, le manque d'information peut entretenir certaines illusions paradisiaques sur les pays de destination potentiels et ainsi favoriser des départs aventureux voire l'exploitation des migrants par des passeurs (Berninghaus et Seifert-Vogt, 1987 ; Allen et Eaton, 2005). Une telle conception - l'information freine la migration - sous-tend les campagnes d'information récurrentes menées par des organisations internationales et par certains pays dans l'espoir d'infléchir des flux migratoires jugés indésirables (Pécoud et Nieuwenhuys, 2008).

Étudiant le lien entre information et caractéristiques individuelles, Gordon F. De Jong et James T. Fawcett rappellent par ailleurs que l'accès à l'information, la possibilité de l'utiliser et la manière de l'interpréter varient au sein des populations (1981). Ces aptitudes dépendent en particulier du niveau de formation, des ressources économiques, de certaines caractéristiques psychologiques telles que l'attitude face à la prise de risque, etc. Elles ont des conséquences directes sur la propension à migrer des individus.

\section{La notion de " place utility " et de " residential stress "}

C'est dans le contexte général de la géographie d'inspiration behavioriste vue comme une alternative aux modèles néo-classiques - que Julian Wolpert développe le concept de "place utility " (1965; Lieber, 1978). Ce dernier se distingue de la simple somme des utilités locales postulées dans le modèle néo-classique en ce qu'il est propre à chaque individu et intègre donc une part de subjectivité liée à des dimensions non nécessairement économiques. Dans le contexte du vocabulaire de l'époque, Wolpert considère les migrations comme résultant d'une série de facteurs de "stress ". Les théories de la satisfaction résidentielle $n^{\prime}$ ont, selon lui, pas assez pris en considération les caractéristiques spécifiques qui lient les personnes à l'endroit où elles habitent et génèrent ou atténuent le niveau de "stress résidentiel " (la somme des facteurs de tous ordres qui contribuent à une éventuelle insatisfaction) (Deane, 1990). Cet élargissement conceptuel consiste en somme à mieux prendre en considération les caractéristiques des lieux de départ et d'arrivée et leur appréhension par les individus. II peut être considéré comme issu des modèles push-pull et des opportunités intermédiaires proposés respectivement par Samuel Stouffer $(1940 ; 1960)$ et par Lee (1966) dans les années 1940 à 1960.

Le concept de "place utilty " joue, aujourd'hui encore, un rôle important dans beaucoup d'études sur les processus de décision migratoire. Ainsi Michael Irwin appelle récemment à étudier " [...] l'effet de la communauté locale sur le 
comportement des individus "(2004-2005 : 654). À partir de cinq études empiriques, Alan Simmons appuie aussi l'intérêt du concept tout en appelant à le combiner avec d'autres variables explicatives (1985-1986). L'étude de Gordon F. De Jong et al. (1985) est plus critique : si elle formule bien l'hypothèse d'un effet du contexte local, elle ne la valide pas dans ses résultats car les liens familiaux, la pression familiale, les cycles de vie et les ressources économiques pèsent en fin de compte plus lourd dans la décision de migrer. Ce résultat peut s'expliquer par le fait, relevé par Thomas Mc Devitt et Saad Gadalla (1985-1986), que les perceptions du contexte peuvent varier - sans doute de manière plus marquée que les autres critères de décisions plus objectifs - au sein d'une unité décisionnelle telle que le ménage. Il en découle la nécessité de prendre en considération les différents membres séparément et surtout de théoriser le processus de négociation et d'arbitrage qui débouche sur la volonté de migrer.

\section{La Nouvelle économie des Migrations (NEM)}

Les années 1990 ont été marquées par un renouveau de la littérature théorique sur les migrations qui a conduit à un élargissement significatif du cadre conceptuel issu de l'école néo-classique. On a en effet assisté, sous le label général de Nouvelle économie des Migrations à un changement de l'acteur de référence (de l'individu au ménage), de l'objectif associé à la migration (de la maximisation du profit à la minimisation du risque) et des critères d'évaluation des conséquences de la migration par les migrants potentiels (d'une valorisation absolue à une valorisation relative).

\section{Le ménage comme unité de prise de décision}

Alors que les écoles précédentes envisageaient la prise de décision à une échelle individuelle, la NEM propose de replacer l'acteur dans le contexte décisionnel plus large du foyer, ou même de la communauté de référence. Les coûts et bénéfices, ainsi que les probabilités de succès ou d'échec, doivent dès lors être calculés pour l'ensemble du ménage. La prise en compte de la structure familiale devient très importante : la taille de la famille, l'âge, le sexe et l'étape dans le cycle de vie des membres mais aussi les modèles de parenté (famille nucléaire ou élargie, etc.) influencent la disposition, les motifs et les attentes face à la migration (Harbinson, 1981). La probabilité de migration peut ainsi différer en fonction du rang dans la fratrie, l'aîné pouvant par exemple être appelé à rester au pays pour se marier et assumer le rôle de chef de famille tandis que son cadet entreprendra une migration.

\section{La migration comme stratégie de gestion des risques}

Le courant néo-classique a traditionnellement considéré l'attitude individuelle face au risque (" aversion au risque " versus " propension au risque ") comme un déterminant de la décision de migrer et le risque d'échec comme influençant I'utilité attendue de la migration (notion d' " espérance mathématique " associée à l'utilité). La NEM ne conteste pas cette relation entre risque et migration mais y ajoute, en quelque sorte, une relation inverse en considérant la migration comme une stratégie de gestion de risque en elle-même. Oded Stark souligne ainsi que dans la perspective d'une rationalité familiale, envoyer un membre du ménage en migration peut s'apparenter à une diversification spatiale du risque plus qu'à une maximisation des rendements économiques espérés (Stark, 1984). 
L'émigration devient une assurance contre les aléas conjoncturels (sécheresse, chômage, etc.), tout particulièrement dans des pays où des solutions d'assurances institutionnelles (assurances des récoltes) n'existent pas. Cet élargissement conceptuel de la notion de risque permet de comprendre pourquoi un fils ou une fille de paysan peut migrer vers la ville ou vers l'étranger même si la probabilité d'y trouver un emploi reste faible et si, de manière générale, sa productivité pourrait être plus élevée sur le domaine familial : avoir un enfant à l'étranger peut ne pas être optimal en période de récoltes " normales " mais vital en cas de crise.

\section{La privation relative}

Oded Stark reprend de Robert Merton le concept de "privation relative " (relative deprivation) et en fait dépendre la satisfaction des individus et leur appréciation des éventuels bénéfices de la migration (Merton, 1957 ; Stark, 1984). La prise en considération de la privation relative permet de conceptualiser la migration dans une perspective de changement social : alors que dans la théorie néo-classique, une certaine quantité de revenu avait une utilité fixe pour un même individu au fil du temps, cette utilité décroît pour la NEM si la situation relative de l'individu au sein de la société se dégrade. Une personne dont la situation ne change pas dans l'absolu (par exemple un fonctionnaire de l'État) peut ainsi être nouvellement incitée à migrer si autour de lui d'autres individus ou groupes sociaux connaissent une amélioration de leur situation économique. Les implications de cet élargissement sont considérables en regard du lien, souvent postulé a priori comme négatif, entre développement économique et émigration. Ce mécanisme pourrait en effet tendre à renforcer - ou au contraire à atténuer si la classe moyenne est plus sensible à la privation relative - la relation "en cloche " (migration hump) souvent observée entre niveau de " développement " et migration (De Haas, 2007).

\section{Les approches issues de la psychologie sociale}

Ce courant est rarement considéré par les synthèses des théories migratoires - il est ainsi ignoré par l'article de référence de Douglas S. Massey et al. de 1993. Ceci s'explique par le fait que la question de la migration n'est abordée que marginalement par les psychologues, dans un contexte plus large de théorisation de la prise de décision. Le point commun de ce courant est la tentative de complexifier le modèle par trop mécaniste du comportement humain implicitement mobilisé par les économistes néoclassiques. On peut mentionner à cet égard les théories de la motivation, les théories des attentes et des valeurs et celles plus spécifiquement développées afin d'appréhender les liens entre attitudes et comportements. Même si, comme nous venons de le relever, ces travaux sont restés relativement en marge de la théorisation des migrations, plusieurs ouvrages initiés par des psychologues ont adopté une démarche interdisciplinaire et synthétique. C'est le cas de deux publications des années 1990. La première est constituée par les actes d'un congrès, édité par Gordon F. De Jong et Robert W. Gardner en 1981, Migration Decision Making. Multidisciplinary Approaches to Microlevel Studies in Developed and Developing Countries. La deuxième est un numéro spécial de la revue Environment and Population des années 1985-1986 entièrement consacré à la prise de décision dans un contexte 
migratoire. Il est assez surprenant de constater que ce thème n'a pas fait l'objet de synthèses plus récentes.

\section{Les théories de la motivation}

Le premier cadre de référence qui a inspiré les études sur le processus décisionnel dans le domaine des migrations est celui de la " prise de décision conflictuelle " développé par Irving L. Janis et Leon Mann (1968). II vise à identifier certaines dimensions socio-psychologiques qui pourraient contribuer à l'explication des différences de comportements des individus face à des pesées d'intérêts conflictuelles tout en se basant sur l'idée que la migration est un acte volontaire et rationnel. Les auteurs différencient ainsi cinq étapes dans la prise de décision :

1. faire face au problème du choix

2. rechercher les différentes possibilités

3. les examiner

4. s'engager dans la décision

5. se tenir à la décision malgré des avis contraires dans la communauté de référence.

Dans ce contexte, c'est pendant longtemps le " deficiency model " qui a prévalu. Ce modèle postule que les personnes qui prennent la décision de migrer ont moins de ressources personnelles et sociales que le reste de la population, sont mal adaptées et souffrent de leur position sociale dans leur pays (Eisenstadt, 1954). Les chercheurs se sont cependant ensuite rendu compte que les migrants disposent souvent, au contraire, de plus de ressources et répondent, plus que les non-migrants, à certains types de motivations. Bonka Boneva et al. (1998) - reprenant de David McClelland (1961) I'idée selon laquelle la motivation humaine est basée sur des objectifs de réussite, de pouvoir ou d'affiliation concluent que les migrants internationaux ont un plus haut niveau de volonté de réussite et de pouvoir et moins de besoins d'affiliation en comparaison des non-migrants. Elles en concluent à l'existence de traits de personnalité spécifiques liés à l'intention de migrer (Boneva et Frieze, 2001). L'indicateur classique du sentiment de maîtrise sur sa propre vie (Locus of control) a été utilisé dans ce contexte avec l'hypothèse - en partie confirmée - qu'il s'avère plus élevé chez les migrants potentiels. Diana B. Winchie met en évidence cet effet dans une étude auprès de 102 hommes indiens ayant l'intention d'émigrer au Canada. Les plus importantes variables distinguant cet échantillon d'un groupe de contrôle de non-migrants sont la satisfaction dans l'emploi, la recherche de sensations, l'intérêt pour le monde extérieur et le locus-of-control (Winchie et Carment, $1988 ; 1989)$.

Par-delà les caractéristiques des individus, des chercheurs se sont intéressé à celles des motivations elles-mêmes. Eugene Tartakovsky et Shalom H. Schwartz (2001) distinguent ainsi trois types de motivations pour émigrer : la préservation (recherche de sécurité), le développement personnel et le matérialisme (amélioration financière). Ils montrent que ces motivations varient et $\mathrm{s}^{\prime}$ articulent avec les valeurs et la personnalité de chaque individu dans un contexte socio-historique donné. 


\section{Les théories des attentes et des valeurs}

La théorie des attentes et des valeurs (value-expectancy) développée par Fishbein (1967) met en place les fondations de nombreux développements des modèles de prise de décision. Elle s'éloigne de l'objectif d'identifier des traits psychologiques favorisant la migration et se penche plutôt sur le processus de décision dans un contexte social donné. Cette théorie postule ainsi un comportement qui se forme en fonction des attentes et des valeurs vis-à-vis du but qu'un acteur se donne. Le comportement choisi sera celui qui a le plus de chances d'obtenir un résultat jugé positif. Gordon F. De Jong et James T. Fawcett (1981) relèvent, à partir d'études empiriques, sept catégories d'attentes pertinentes dans le processus décisionnel migratoire : richesse, statut, confort, stimulation (avoir des activités plaisantes), autonomie, affiliation (rejoindre d'autres personnes) et moralité (croyance sur la bonne manière de vivre). Ils soulignent également que d'autres facteurs influencent la décision de migrer : les traits individuels, les normes sociales et culturelles, la propension à prendre des risques et la structure d'opportunité. Le migrant n'est pas totalement libre de ses choix et des facteurs structuraux interviennent dans sa prise de décision (Haug, 2008). Le genre est fréquemment cité à cet égard comme une variable importante de différenciation. Lu Yixi (2006) constate ainsi une différence sensible entre les étudiants masculins et féminins chinois au Canada. Selon elle, les filles auraient un lien émotionnel plus fort à la famille et un plus grand besoin de sentiment de stabilité dans leur perception des expériences. Les valeurs de la société de départ en regard de la migration doivent en outre être spécifiquement prises en considération (De Jong and Gardner, 1981). La communauté de référence peut en effet soit valoriser, soit condamner ses membres qui entreprennent une migration. L'exemple le plus fréquemment cité est celui de la pression sociale à l'immobilité souvent exercée sur les femmes "[...] social norms regarding gender roles play an important role in promoting or hindering migration by females and males. The patriarchal family system accepts and foments male migration, but hinders female migration. Fathers are more likely to resist the migration of daughters, and husband the accompaniment of their wives and children, even years after first leaving home " (Davis et Winters, $2001: 10)$.

\section{Le modèle de l'action raisonnée et du comportement planifié}

Les approches évoquées jusqu'ici posaient un lien relativement direct entre la migration et un certain nombre de facteurs personnels et contextuels. Le grand intérêt du modèle de comportement planifié (MCP) - issu du modèle de I'action raisonnée (MAR) présenté par Icek Ajzen et Martin Fishbein dans leur ouvrage de 1980, Understanding Attitudes and Predicting Social Behavior - est de distinguer, d'une part, l'intention - influencée par une première série de considérations et de contraintes - d'autre part le comportement proprement dit - potentiellement influencé par d'autres facteurs et contraintes. La question posée est dès lors celle du lien entre les attitudes (par exemple la volonté de partir) et les actions proprement dites (la migration elle-même). Cette question est abordée dans un contexte de migration par une méta-analyse de Christopher Armitage et Mark Conner (2001) ainsi que dans un article de Max Lu précisément intitulé, Do people move when they say they will...? (Lu, 1999). Parmi les facteurs souvent mis en évidence pour expliquer un décalage entre intentions et actions, les auteurs citent l'âge, le genre, le statut du logement, etc. De manière 
générale cependant, plusieurs auteurs soulignent que, dans le domaine des migrations, intentions et actions s'avèrent relativement corrélées ou répondent pour le moins à des facteurs explicatifs semblables. Selon van Dalen, même si les intentions ou les décisions de migrer ne mènent pas nécessairement à l'acte lui-même, on peut considérer qu'un fort lien existe (van Dalen et al., 2005).

\section{Les approches sociologiques et géographiques}

Les théorisations présentées jusqu'ici s'avèrent largement centrées sur les individus. Plusieurs développements relativement récents cherchent à mieux prendre en compte les interrelations entre le migrant et son environnement social, que ce soit en termes de réseaux ou de représentations collectives.

\section{La théorie des réseaux et le capital social}

La prise en compte des liens entre les connexions interpersonnelles et les migrations peut se rattacher aux concepts classiques de chaîne migratoire d'une part (OECD, 1978) de capital social d'autre part (Portes, 1998). Elle a retenu de manière croissante l'attention des chercheurs avec le passage d'une conception dominante de la migration comme une rupture à celle de la migration comme constitutive de liens transnationaux à travers l'espace (Massey, 1990 ; Faist, 1997). L'individu confronté à la décision de migrer est désormais considéré comme relié à une structure sociale constituée par la famille proche et élargie, par les personnes originaires de la même région, du même groupe culturel ou plus largement par des amis et connaissances. Ces réseaux sont à la fois des sources d'informations et des fournisseurs d'aides et d'appui pour le voyage et I'installation dans un pays de destination. On considère en général que l'existence de réseaux facilite la migration (De Jong, 2000). Ainsi, dans le cas du Ghana, Shani Salifu souligne l'importance du réseau social et des réciprocités qu'il implique déjà dans le pays d'origine : " [...] social networks in Ghana played a role in motivating people to travel. More than $20 \%$ of the participants reported that the opportunity to travel was acquired for them by family members [...]. In other words, social networks will facilitate migration with the understanding that the migrant would assist with some of the responsibilities that the network concerned is burdened with "(Salifu, 2007 : 67). Certains réseaux peuvent cependant aussi avoir un effet de frein en diffusant des informations dissipant des illusions sur les perspectives migratoires ou en impliquant des contraintes supplémentaires pour le migrant (Faist, 1997).

Selon Sonja Haug (2008), qui se base sur (Ritchey, 1976), cinq mécanismes expliquent comment les liens communautaires et familiaux influencent la prise de décision : les affinités, l'information, la facilitation, le conflit et l'encouragement. L'affinité que l'on pourrait définir comme le " souhait d'être ensemble " a pour conséquence que des réseaux sociaux sur place découragent la migration tandis que des réseaux sociaux ailleurs l'encouragent. Ces mêmes réseaux à l'étranger favorisent la collecte d'informations sur le pays mais facilitent également le déplacement en prenant en charge le nouveau migrant à son arrivée. Si des conflits internes à certains réseaux - familiaux par exemple existent dans le pays de résidence, ils pousseront au départ, de même que des normes familiales ou communautaires favorisant la migration. Hendrik P. Van Dalen et al. (2005) suggèrent en outre que l'impact des réseaux s'avère parti- 
culièrement crucial précisément au stade préliminaire du processus migratoire c'est-à-dire lors de la formation d'un souhait ou d'une intention.

Deux concepts connexes peuvent être mentionnés ici : celui de l'effet cumulatif (cumulative causation) qui implique que la migration internationale s'auto-entretient, entre autres précisément par le biais des réseaux qu'elle met en place et du changement social qu'elle implique sur le contexte de départ (Massey et al., 1993) et celui des canaux migratoires (migration channels) qui souligne la mise en place au fil du temps de trajectoires migratoires à travers l'espace reliant de manière privilégiée certains lieux de départ et d'arrivée et corrélatives du développement plus large des réseaux (Findlay, 1990). Ces concepts sont importants pour expliquer le maintien du phénomène migratoire même si les facteurs ayant conduit à son émergence cessent d'avoir un effet (par exemple si le différentiel de salaires se comble). Il est aussi important pour expliquer le retour ou le non-retour des migrants : selon la vision dominante, le capital social dans le pays d'origine tend à décliner au fil du temps, alors qu'il s'accroit dans le pays d'accueil ce qui n'encourage pas les migrants au retour. Cette conception de la migration est cependant mise en question par des approches plus récentes qui mettent l'accent sur le potentiel des moyens de communication (Internet, Skype, téléphonie mobile, etc.) à faciliter le maintien d'un capital social et de réseaux transnationaux. Il en découlerait une intensification de la circulation à double sens entre espace de départ et d'arrivée, voire avec d'autres espaces de transit (Glick-Schiller et al., 1995).

Un certain nombre de critiques peuvent être adressées aux approches en termes de réseaux. Ainsi, les études empiriques adoptant cette approche se focalisent souvent étroitement sur la famille en tant qu'unité décisionnelle de référence (Fawcett et Arnold, 1987 ; Root et De Jong, 1991 ; Kulu-Glasgow, 1992 ; Palloni et al., 2001 ; Herman, 2006). Certaines études ont par ailleurs désigné les réseaux comme le facteur d'explication dominant des migrations au détriment de facteurs structurels tels que la demande du marché du travail ce qui leur a valu des critiques acerbes (Krissman, 2005). De nombreux auteurs s'accordent pourtant à relever l'apport significatif de la théorie des réseaux et le fait qu'une conception étendue de cette notion et une différenciation typologique, dans la lignée entre autres des travaux classiques sur les effets respectifs des liens faibles et des liens forts de Granovetter (1983), permettrait d'affiner considérablement la compréhension de la prise de décision migratoire.

\section{L'imaginaire géographique}

Si la notion de "place utility " évoquée ci-dessus était encore fortement chargée de l'idée comportementaliste d'une réaction migratoire mécanique à un certain nombre d'attributs locaux plus ou moins subjectivement appréhendés par les acteurs, le tournant culturel en sciences sociales permet de suggérer des conceptualisations beaucoup plus larges des motifs de migration et du choix des destinations, en particulier autour de la notion d'imaginaire géographique. En suivant la remarque d'Arjun Appadurai selon lequel "Imagination is now central to all forms of agency, is itself a social fact, and is the key component of the new global order "(Appadurai, 1996 : 31), on peut en effet considérer qu'entrent en jeu dans la décision de migrer, en plus des différents facteurs énumérés jusqu'ici, de grands schémas stéréotypiques d'interprétation du 
monde, propres soit à certaines périodes historiques et à certaines "cultures" dans la lignée de I'Orientalisme d'Edward Saïd (Gregory, 1995), soit à certains individus en raison de leur expérience singulière du monde dans un contexte de circulation grandissante d'images, de médias et d'idéologies. L'intérêt des géographes pour la manière dont les individus perçoivent l'espace n'est pas nouveau et remonte au courant des "cartes mentales " qui s'est développé dans les années 1960 pour rendre compte du décalage cognitif entre perceptions et réalité de l'espace (Gould et White, 1984). Les démarches contemporaines dépassent cependant cette distinction et prennent en considération le caractère performatif de l'imaginaire géographique, ses enjeux identitaires et les rapports de pouvoir qui le sous-tendent. Selon ce courant, s'ils veulent comprendre les motivations migratoires des individus dans toute leur complexité, les chercheurs se doivent de prendre en compte ces aspects tout particulièrement s'ils veulent saisir la manière dont les migrants potentiels procèdent à une hiérarchisation des destinations possibles.

Yvonne Riaño et Nadia Baghdadi donnent l'exemple d'une telle analyse au sujet des migrations de mariages. Elles montrent à quel point, dans le cas de la Suisse et de l'Amérique du Sud, I'image des deux espaces respectifs influence le choix du lieu d'établissement de couples mixtes (Riaño et Baghdadi, 2007). Le récit qui accompagne les aspirations migratoires doit dans ce contexte être recueilli directement auprès des acteurs et mis en parallèle avec d'autres récits véhiculés, par exemple, par les médias ou les autorités d'émigration ou d'immigration. De même, il y a lieu de saisir la géographie imaginaire des pays de destination possible et d'en comprendre les facteurs sous-jacents. Ainsi que le montre Jana Sladkova (2007), cette attention portée à la narration ne débouche pas sur la mise en lumière de schémas explicatifs radicalement différents de ceux postulés par les théories dominantes du domaine des migrations - ainsi la primauté des motifs économiques demeure en général - mais elle permet néanmoins d'affiner considérablement la compréhension du sens qu'y accordent les acteurs concernés.

Luc Sindjoun tout comme Jean-Marc Ela et Anne-Sidonie Zoa relèvent à ce sujet, pour le cas africain, que la migration n'est de loin pas toujours un projet résultant de la seule compilation de toutes les informations objectives. Les décisions de migrer et l'option pour une destination sont souvent conditionnées par l'expérience des aînés du clan, la familiarité culturelle, la mémoire de la migration enracinée dans I'histoire, la tradition initiatique propre aux sociétés africaines, l'imaginaire social renouvelé qui caractérise les nouvelles générations etc. (Sindjoun, 2004 ; Ela et Zoa, 2006). Ces considérations interviennent comme une grille de lecture complémentaire - et non pas alternative - aux théories existantes pour comprendre les nouveaux enjeux des migrations et les motifs de départ ou de non-départ des individus.

\section{Conclusion}

Notre tour d'horizon permet d'identifier les principales propositions théoriques issues de la littérature scientifique pour comprendre la migration à l'échelle individuelle. Au fil des travaux évoqués un consensus se dégage pour mettre en avant le caractère multidimensionnel du phénomène migratoire. II est 
rare en effet qu'un individu s'engage dans la migration en raison d'un facteur unique tel que le différentiel de salaire ou de chômage (comme le suppose la théorie néo-classique), en raison de traits de personnalités spécifiques (comme l'ont postulé les premières théories psychologiques) ou encore de différences dans l'utilité escomptée (" place utility ") entre lieu de départ et d'arrivée (comme l'envisageaient les approches comportementales des années 1960). II est reconnu désormais que des considérations familiales et politiques, des réseaux sociaux, voire des stéréotypes ou un imaginaire géographique interagissent de manière complexe dans un processus de choix qui s'effectue toujours sous contrainte (Lu, 1999 : 486) et aboutit à la migration ou à la non-migration. Comme le relève Babacar Ndione : "II est aujourd'hui admis que la volonté et la capacité de migrer à l'étranger résultent à la fois de la personnalité et des trajectoires socio-économiques du candidat migrant, de l'environnement produit par son ménage et sa communauté de référence, des circuits d'information auxquels il est exposé, des réseaux migratoires et des contextes politiques et économiques du pays d'accueil "(Ndione, $2008: 2$ ). Ceci ne revient pas à considérer que l'on migre pour toutes sortes de raisons et que le phénomène ne serait en fin de compte pas susceptible d'être théorisé, comme le pensaient d'ailleurs au XIXe siècle certains des contradicteurs oubliés de Ravenstein. II s'agit plutôt de rejoindre l'option du pluralisme théorique suggérée par Douglas S.Massey et al., il y a deux décennies (Massey et al., 1993 ; 1994). L'idée est de procéder en deux temps ; mobiliser conjointement des facteurs explicatifs issus de plusieurs traditions disciplinaires afin de proposer un système d'hypothèses, puis pondérer les différents facteurs par des investigations empiriques. Le schéma ci-dessous (Figure 1) présente, à titre d'exemple illustratif, le système d'hypothèses retenu dans une récente recherche en Afrique de l'Ouest. L'effet des différents facteurs ne sera pas simplement additif mais découlera de combinaisons contextuelles. Ainsi, parmi les étudiants ivoiriens, nigériens et sénégalais, les intentions migratoires vont dépendre du soutien de la famille et de l'existence d'un réseau mais varier simultanément en fonction de l'image que se fait chaque étudiant des perspectives futures de son pays en termes économiques et politiques (Piguet et Efionayi, 2011). Certaines conditions sont nécessaires sans être suffisantes pour déclencher le départ, des effets de seuil se manifestent et débouchent sur des relations non linéaires. Ainsi, les relations entre le revenu du ménage et la propension au départ peuvent adopter un profil " en cloche " quand seuls ceux qui atteignent un certain niveau de revenu - ni les plus pauvres, ni les plus riches - envisagent le départ. Le pluralisme théorique permet aussi de rendre compte de l'évolution temporelle des phénomènes migratoires et de leur caractère auto-entretenu ou cyclique. Ainsi les facteurs explicatifs de la phase initiale de migration peuvent se rapprocher de la théorie néo-classique du différentiel économique, mais les réseaux constitués au fil du temps peuvent ensuite se substituer aux variables économiques et assurer la perpétuation du phénomène même si une convergence économique des espaces de départ et d'arrivée se manifeste entre temps. Des réseaux en sommeil peuvent aussi être réactivés et expliquer conjointement avec d'autres facteurs la reprise de certains flux migratoires. Ainsi la résurgence très récente d'une migration en provenance du sud de l'Europe vers certaines régions suisses renvoie à la fois aux politiques de recrutement helvétiques des années 1960 et à la crise économique qui frappe actuellement des pays comme I’Espagne, I'Italie ou le Portugal (Piguet, 2013). 
Figure 1

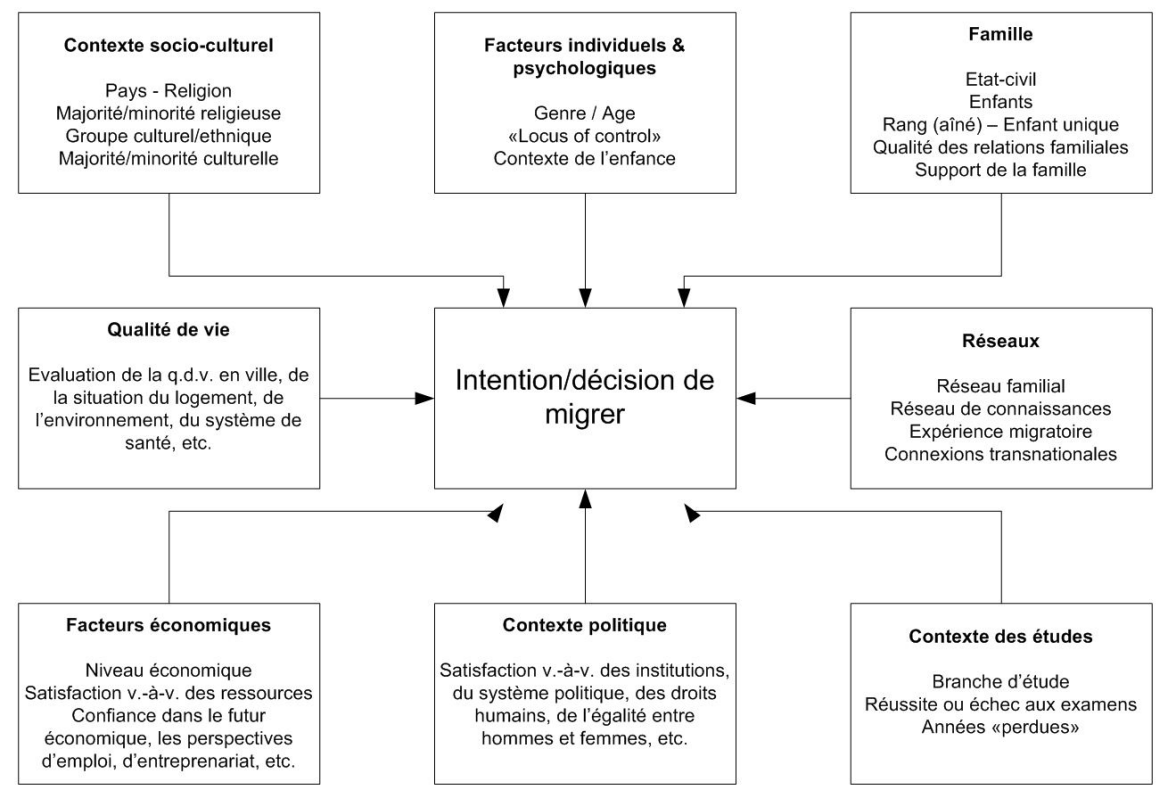

L'option du pluralisme théorique présente évidemment moins de cohérence d'ensemble qu'une approche se référant strictement à un courant spécifique, mais elle s'avère plus souple pour rendre compte de la complexité et de la diversité des migrations contemporaines. Elle se rattache par ailleurs aux propositions théoriques qui, depuis plusieurs années déjà, visent à prendre en compte simultanément les dimensions structurelles qui restreignent les choix des individus et la marge d'action (agency), de créativité ou même d'imagination qu'ils parviennent à se ménager (Bakewell, 2010 ; Smith and King, 2012). Elle donne une visée à la recherche empirique : il va s'agir, par des méthodes qualitatives, quantitatives ou combinées (Foner et al., 2003) de produire des études de cas suffisamment comparables pour susciter un processus cumulatif de connaissance (Brettell et Hollifield, 2007 ; Favell, 2007). Pour qu'un tel objectif soit atteint, il sera cependant aussi nécessaire de faire le pont entre différentes échelles et c'est là certainement que nous atteignons les limites de la présente synthèse. Ainsi, si nous avons pu montrer en quoi le passage de l'individu au ménage et du ménage au réseau était fécond, nous n'avons - faute de place rendu compte ni des théorisations à échelle macro des migrations proposées par des courants aussi variés que la World system theory, la théorie du marché du travail dual ou la théorie néo-classique (Massey et al., 1993), ni des théories visant indirectement à expliquer les flux migratoires à travers une meilleure compréhension des politiques qui les conditionnent (Meyers, 2000). Pour Money et Zartner Fastrom : "Much of the literature addresses the determinants of flows from the perspective of individual migrants and the firms that employ migrants, leaving aside the policy choices of states that govern those flows or, at best, placing these factors in the equation as cost to migrants and employers " (Money et Zartner Fastrom, 2006 : 136). Là aussi, il s'agira de prendre en 
compte les interactions et les complémentarités entre les échelles. Comme le relève Massey "The various explanations are not necessarily contradictory [...] it is entirely possible that individuals engage in cost-benefit calculations; that houselholds act to diversify labor allocations; and that the socioeconomic context within which these decisions are made is determined by structural forces operating at the national and international levels " (Massey et al., 1993: 454-455). Mais ceci devra faire l'objet d'un autre article.

\section{Références bibliographiques}

Allen Jeremiah M. and Eaton B. Curtis (2005) Incomplete Information and Migration: the Grass is Greener across the Higher Fence, Journal of Regional Science, 45 (1), pp. 1-19.

Appadurai Arjun (1996) Modernity at Large: Cultural Dimensions of Globalization, Minneapolis, University of Minnesota Press, 248 p.

Arango Joaquim (2000) Explaining Migration: A Critical View, International Social Science Journal, 52, pp. 283-296.

Armitage Christopher J. and Conner Mark (2001) Efficacy of the theory of planned behavior: A meta-analytic review, British Journal of Social Psychology, 40, pp. 471-499.

Bakewell Oliver (2010) Some Reflections on Structure and Agency in Migration Theory, Journal of Ethnic and Migration Studies, 36 (10), pp. 1689-1708.

Berninghaus Siegfried and Seiffert-Vogt Hans-Günther (1987) International migration under incomplete information, Schweizerische Zeitschrift für Volkswirtschaft und Statistik, 123 (2), pp. 199-218.

Berthomière William et Hily Marie-Antoinette (2006) Décrire les migrations internationales. Les expériences de la co-présence, Revue Européenne des Migrations Internationales, 22 (2), pp. 67-82.

Boneva Bonka and Frieze Irene (2001) Toward a Concept of Migrant Personality, Journal of Social Issues, 57 (3), pp. 477-491.

Boneva Bonka, Frieze Irene, Ferligoj Anuska, Jarosova Eva, Pauknerova Daniela and Orgocka Aida (1998) Achievement, Power, and Affiliation Motives as Clues to (E)migration Desires: A Four-Countries Comparison, European Psychologist, 3 (4), pp. 247-254.

Boyle Paul J., Halfacree Keith and Robinson Vaughan (1998) Exploring Contemporary Migration, London, Longman, 296 p.

Brettell Caroline B. and Hollifield James F. (Eds.) (2007) Migration theory: Talking across disciplines, London, Routledge, $304 \mathrm{p}$.

Chiswick Barry R. (2007) Are Immigrants Favorably Self-Selected?, in Caroline B. Brettell and James F. Hollifield Eds., Migration Theory. Talking across Disciplines, London, Taylor \& Francis, pp. 63-82.

Cohen Robin (Ed.) (1996) Theories of migration, Cheltenham, E. Elgar, 512 p.

Courgeau Daniel et Lelièvre Éva (2003) Les motifs individuels et sociaux des migrants, in Graziella Caselli, Jacques Vallin et Guillaume Wunsch Éds., Les déterminants de la migration, Paris, INED, pp. 147-169. 
Da Vanzo Julie (1981) Microeconomic Approaches to Studying Migration Decisions, in Gordon F. De Jong and Robert W. Gardner Eds., Migration Decision Making. Multidisciplinary Approaches to Microlevel Studies in Developed and Developing Countries, New York/Oxford, Pergamon Press, pp. 90-129.

Davis Benjamin and Winters Paul (2001) Gender, Networks and Mexico-US Migration, Journal of Development Studies, 38 (2), pp. 1-26.

De Haas Hein (2007) Le mythe de l'invasion. Migration irrégulière d'Afrique de I'Ouest au Maghreb et en Union européenne, Oxford, International Migration Institute (IMI), $81 \mathrm{p}$.

De Jong Gordon F. (2000) Expectations, Gender, and Norms in Migration Decision-making, Population Studies, 54 (3), pp. 307-319.

De Jong Gordon F. and Fawcett James T. (1981) Motivations for migration: An assessment and a value-expectancy research model, in Gordon F. De Jong and Robert W. Gardner Eds., Migration decision making: Multidisciplinary approaches to microlevel studies in developed and developing countries, New York, Pergamon Press, pp. 13-58.

De Jong Gordon F. and Gardner Robert W. (Eds.) (1981) Migration decision making: Multidisciplinary approaches to microlevel studies in developed and developing countries, New York, Pergamon, 394 p.

De Jong Gordon F., Root Brenda Davis, Gardner Robert W., Fawcett James T. and Abad Ricardo G. (1985) Migration Intentions and Behavior: Decision Making in a Rural Philippine Province, Population and Environment, 8 (1/2), pp. 41-61.

Dane Glenn D. (1990) Mobility and adjustments: paths to the resolution of residential stress, Demography, 27 (1), pp. 65-79.

Eisenstadt Shmuel Noah (1954) The absorption of immigrants, London, Routledge, $275 \mathrm{p}$.

Ela Jean-Marc et Zoa Anne-Sidonie (2006) Fécondité et migrations africaines : les nouveaux enjeux, Paris, L'Harmattan, $356 \mathrm{p}$.

FaistThomas (1997)The Crucial Meso Level, inThomas Hammar Eds., International Migration, Immobility and Development: Multidisciplinary Perspectives, Oxford: Berg, pp. 47-64.

Favell Adrian (2007) Rebooting migration theory: interdisciplinarity, globality and postdisciplinarity in migration studies, in Caroline B. Brettell and James F. Hollifield Eds., Migration Theory: Talking across Disciplines, New York, Routledge, pp. 259-278.

Fawcett James T. and Arnold Fred (1987) The Role of Surveys in the Study of International Migration: An Appraisal, International Migration Review, 21 (4), pp. 1523-1540.

Findlay Allan (1990) A Migration Channels Approach to the Study of High Level Manpower Movements: A Theoretical Perspective, International Migration, 28 (1), pp. 15-23.

Fischer Peter A., Martin Reiner and Straubhaar Thomas (1997) Should I Stay or Should I Go?, in Tomas Hammar, Grete Brochmann, Kristof Tamas and Thomas Faist Eds., International migration, immobility and development: multidisciplinary perspectives, Oxford Berg, pp. 49-90. 
Fishbein Martin (1967) Attitude and the prediction of behavior, in Martin Fishbein Ed., Readings in attitude theory and measurement, New York, Wiley, pp. 477-492.

Foner Nancy, Rumbaut Rubén G. and Gold Steven J. (Eds.) (2003) Immigration research for a new century: multidisciplinary perspectives, New York, Russell Sage Foundation, 491 p.

Geyer Hermanus S. (2002) An exploration in migration theory, in Hermanus S. Geyer Ed., International handbook of urban systems: Studies of urbanization and migration in advanced and developing countries, Cheltenham, Edward Elgar, pp. 19-37.

Ghatak Subrata, Levine Paul and Price Stephen Wheatley (1996) Migration theories and evidence: An assessment, Journal of Economic Surveys, 10 (2), pp. 159-198.

Gould Peter et White Rodney (1984) Cartes Mentales, Fribourg, Éditions Universitaires [première publication en langue anglaise, 1974], 188 p.

Granovetter Mark J. (1983) The Strength of Weak Ties: A Network Theory Revisited, SociologicalTheory, 1, pp. 201-233.

Gregory Derek (1995) Imaginative geographies, Progress in Human Geography, 19 (4), pp. 447-485.

Harbinson Sarah (1981) Family Structure and Family Strategy in Migration Decision Making, in Gordon F. De Jong and Robert W. Gardner Eds., Migration Decision Making. Multidisciplinary Approaches to Microlevel Studies in Developed and Developing Countries, New York, Pergamon Press, pp. 225-251.

Harris John R. and Todaro Michael P. (1970) Migration, unemployment and development: A two-sector analysis, American Economic Review, 60 (1), pp. 126-142.

Haug Sonja (2008) Migration Networks and Migration Decision-Making, Journal of Ethnic and Migration Studies, 34 (4), pp. 585-605.

Herman Emma (2006) Migration as a family business: The role of personal networks in the mobility phase of migration, International Migration, 44 (4), pp. 191-230.

Homans George C. (1961) Social Behaviour: Its Elementary Forms, London, Routledge and Kegan Paul, 407 p.

Irwin Michael, Blanchard Troy, Tolbert Charles, Nucci Alfred et Lyson Thomas (2004-2005) Pourquoi certains ne migrent pas : l'impact du contexte local sur la sédentarité aux États-Unis, Population, 59, pp. 653-680.

Janis Irving L. and Mann Leon (1968) Decision Making: A Psychological Analysis of Conflict, Choice and Commitment, New York, Free Press, 488 p.

Krissman Fred (2005) Sin Coyote Ni Patron: Why the "Migrant Network" Fails to Explain International Migration, International Migration Review, 39 (1), pp. 4-44.

Kulu-Glasgow Isik (1992) Motives and social networks of international migration within the context of the systems approach: a literature review, Den Haag, Netherlands Interdisciplinary Demographic Institute, $71 \mathrm{p}$.

Lee Everett (1966) A theory of migration, Demography, 3 (48), pp. 47-57.

Leslie Gerald R. and Richardson Arthur H. (1961) Life-Cycle, Career Pattern, and the Decision to Move, American Sociological Review, 26 (6), pp. 894-902. 
Lieber Stanley R. (1978) Place Utility and Migration, Geografiska Annaler. Series B, Human Geography, 60 (1), pp. 16-27.

Lu Max (1999) Do People Move When They Say They Will? Inconsistencies in Individual Migration Behavior, Population \& Environment, 20 (5), pp. 467-488.

Ma Mung Emmanuel, Dorai Kamel, Hily Marie-Antoinette et Loyer Franz (1998) Bilan des travaux sur la circulation migratoire, Migrations Études, 84, pp. 1-12.

Massey Douglas S. (1990) The social and economic origins of immigration, Annals of the American Academy of Political and Social Sciences, 510, pp. 60-72.

Massey Douglas S., Arango Joaquin, Hugo Graeme, Kouaouci Ali, Pellegrino Adela and Taylor J. Edward (1994) An Evaluation of International Migration Theory: The North American Case, Population and Development Review, 20 (4), pp. 699-751.

Massey Douglas S., Arango Joaquin, Hugo Graeme, Kouaouci Ali, Pellegrino Adela and Taylor J. Edward (1993) Theories of international migration: A review and appraisal, Population and Development Review, 19 (3), pp. 431-466.

McDevitt Thomas M. and Gadalla Saad M. (1985-1986) Incorporating HusbandWife Differences in Place Utility Differentials into Migration Decision Models, Population and Environment, 8 (1-2), pp. 98-119.

McClelland David (1961) The Achieving Society, Toronto, Princeton New Jersey, D. Van Nostrand, 530 p.

Merton Robert K. (1957) Social theory and social structures, Glencoe, Free Press, $702 \mathrm{p}$.

Meyers Eytan (2000) Theories of International Immigration Policy. A Comparative Analysis, International Migration Review, 34 (4), pp. 1245-1282.

Money Jeannette and Zartner Falstrom Dana (2006) Interests and Institutions in Skilled Migration: Comparing Flows in the IT and Nursing Sectors in the US, in Smith Michael Peter and Favell Adrian Eds., The human face of global mobility: international highly skilled migration in Europe, North America and Asia-Pacific, New Brunswick, Transaction Publishers, pp. 131-158.

Ndione Babacar (2008) Contexte local et migration : I'exemple des dynamiques migratoires internationales des quartiers dans la ville sénégalaise de Kaolack, European Journal of Population, 25 (3), pp. 325-354.

Noin Daniel (2005) Géographie de la population, Paris, Armand Colin, 280 p.

Odland John (1988) Migration and occupational choice among young labor force entrants: a human capital model, Geographical analysis, 20 (4), pp. 281-296.

OECD (1978) The migratory chain, Paris, Organisation for economic cooperation and development, $53 \mathrm{p}$.

Palloni Alberto, Massey Douglas S., Ceballos Miguel, Espinosa Kristin and Spittel Michael (2001) Social Capital and International Migration: A Test Using Information on Family Networks, American Journal of Sociology, 106 (5), pp. 1262-1298.

Pécoud Antoine et Nieuwenhuys Céline (2008) Campagnes d'information et traite des êtres humains à l'est de l'Europe, Espace Populations Sociétés, 2, pp. 319-330. 
Piguet Étienne (2013) L'immigration en Suisse. Soixante ans d'entrouverture, Lausanne, Presses polytechniques romandes (3 $3^{\mathrm{ème}}$ édition mise à jour), $147 \mathrm{p}$.

Piguet Étienne et Efionayi Denise (2011) Partir ou rester ? La migration dans le projet de vie des étudiants universitaires d'Afrique de l'Ouest, Geneva, Swiss Network For International Studies (Research Report), 85 p.

Portes Alejandro (1998) Social Capital: Its Origin and Applications in Modern Sociology, Annual Review of Sociology, 24, pp. 1-24.

Portes Alejandro and Böröcz József (1996) Contemporary immigration: Theoretical perspectives on its determinants and modes of incorporation, International Migration Review, 23 (3), pp. 606-630.

Ratzel Friederich (1882) Anthropogeographie (Anthropogeography), Stuttgart, Engelhorn, $506 \mathrm{p}$.

Ravenstein Ernst G. (1889) The laws of migration, Journal of the Royal Statistical Society, 52 (2), pp. 241-305.

Rérat Patrick, Piguet Etienne, Besson Roger et Söderström Ola (2008) Les âges de la ville. Mobilité résidentielle, parcours de vie et attractivité des villes suisses, Geographica helvetica, 4, pp. 261-271.

Riaño Yvonne et Baghdadi Nadia (2007) Je pensais que je pourrais avoir une relation plus égalitaire avec un Européen. Le rôle du genre et des imaginaires géographiques dans la migration des femmes, Nouvelles Questions Féministes, 1, pp. 38-53.

Ritchey Neal (1976) Explanations of migration, Annual Review of Sociology, 2, pp. 363-404.

Root Brenda Davis and DE Jong Gordon F. (1991) Family Migration in a Developing Country, Population Studies, 45 (2), pp. 221-233.

Rossi Peter Henry (1955) Why families move? A study in the social psychology of urban residential mobility, Glencoe, The Free Press, 220 p.

Salifu Shani (2007) Factors Contributing to Migration from Ghana to the United States of America, Master of Arts - Ohio University - Center for International Studies, $127 \mathrm{p}$.

Simon Herbert (1955) A Behavioral Model of Rational Choice, The Quarterly Journal of Economics, 69 (1), pp. 99-118.

Simon Herbert (1957) Models of Man: Social and Rational, New York, Wiley, $279 \mathrm{p}$.

Sindjoun Luc (2004) État, individus et réseaux dans les migrations africaines, Paris, Karthala, 360 p.

Sjaastad Larry A. (1962) The Costs and Returns of Human Migration, Journal of Political Economy, 70, pp. 80-93.

Sladkova Jana (2007) Expectations and Motivations of Hondurans Migrating to the United States, Journal of Community \& Applied Social Psychology, 17, pp. 187-202.

Smith Adam (1776) La richesse des nations (tomes 1 et 2), Paris, Flammarion, $1168 \mathrm{p}$. 
Smith Darren P. and King Russell (2012) Editorial Introduction: Re-Making Migration Theory, Population, Space and Place, 18 (2), pp. 127-133.

Stark Oded (1984) Discontinuity and the theory of international migration, Kyklos, 37 (2), pp. 206-222.

Stouffer Samuel (1960) Intervening opportunities and competing migrants, Journal of Regional Science, 2 (1), pp. 1-26.

Stouffer Samuel (1940) Intervening opportunities: A theory relating mobility and distance, American Sociological Review, 5 (6), pp. 845-867.

Tapinos Georges Photios (1974) L'économie des migrations internationales, Paris, Armand Colin, 287 p.

Tarrius Alain (1989) Anthropologie du mouvement, Caen, Paradigme, 192 p.

Tartarovsky Eugene and Schwartz Shalom H. (2001) Motivation for emigration, values, wellbeing, and identification among young Russian Jews, International Journal of Psychology, 36 (2), pp. 88-99.

Uhlenberg Peter (1973) Noneconomic Determinants of Nonmigration: Social Considerations for Migration Theory, Rural Sociology, 67 (1), pp. 296-311.

Van Dalen Hendrik P., Groenewold George and Schoorl Jeannette J. (2005) Out of Africa: What Drives the Pressure to Emigrate?, Journal of Population Economics, 18 (4), pp. 741-778.

Winchie Diana B. and Carment David W. (1989) Migration and Motivation: The Migrant's Perspective, International Migration Review, 23 (1), pp. 96-104.

Winchie Diana B. and Carment David W. (1988) Intention to Migrate: A Psychological Analysis, Journal of Applied Social Psychology, 18 (9), pp. 727-736.

Wolpert Julian (1965) Behavioral aspects of the decision to migrate, Papers of the Regional Science Association, 15 (1), pp. 159-169.

Yixi Lu (2006) Studying Abroad and Migration Motivations: A Case Study of Chinese Students at the University of Saskachewan, Canada, Saskatoon, University of Saskatchewan, 175 p.

Zlotnik Hania (2003) Théories sur les migrations internationales, in Graziella Caselli, Jacques Vallin et Guillaume Wunsch Éds., Les Déterminants de la migration, Paris, Institut National d'Études Démographiques, pp. 55-78.

Zolberg Aristide R. (1989)The next waves: Migration theory for a changing world, International Migration Review, 23 (3), pp. 403-430. 


\section{Étienne Piguet}

\section{Les théories des migrations. Synthèse de la prise de décision individuelle}

Cet article propose une synthèse théorique au sujet de la prise de décision migratoire en mentionnant les principaux travaux de référence. Nous tentons de brosser un tableau intégrant aussi bien les approches des géographes, démographes, économistes et sociologues que celles issues de la psychologie sociale ou de l'anthropologie. L'originalité de la présente démarche est de faire converger ces corpus souvent distincts et de combler une lacune : on ne dispose pas de synthèse récente sur les théories migratoires.

\section{.... Theories of Migration. Synthesis of Individual Decision-making}

This paper offers an overview of the theorization of migration decision making. We give a general picture which includes geographic, demographic, economic and sociological approaches as well as theories coming from social psychology and anthropology. The originality of the paper is that it attempts at presenting in a coherent way scientific corpuses usually dealt with in isolation. It also answers to the need of a contemporary synthesis about migration theory that is currently missing.

\section{Las teorías de las migraciones. Síntesis de la toma de decisiones individual}

Este artículo propone una síntesis teórica respecto a la temática de la toma de decisión migratoria y presenta los trabajos principales de referencia. El esfuerzo principal consiste en bosquejar un cuadro que integra tanto los enfoques de los geógrafos, los demógrafos, los economistas y los sociólogos como aquellos que surgen de la sicología social o la antropología. La originalidad del enfoque presentado consiste, de una parte, en hacer confluir enfoques diversos, y de otra, de llenar una laguna de conocimiento ya que actualmente no disponemos de una síntesis reciente sobre teorías migratorias. 